\title{
The Higgs field as an inflaton
}

\author{
Fedor Bezrukov \\ Physics Department, University of Connecticut, Storrs, CT 06269-3046, USA, \\ RIKEN-BNL Research Center, Brookhaven National Laboratory, Upton, NY 11973-5000, \\ USA \\ E-mail: Fedor.Bezrukov@uconn.edu
}

\begin{abstract}
The Higgs field of the pure Standard Model can lead to the inflationary expansion of the early Universe if it is non-minimally coupled to gravity. The model predicts Cosmic Microwave Background (CMB) parameters in perfect agreement with the current observations and has implications for the Higgs boson mass. We review the model, its predictions, problems arising with its quantization and some closely related models.

PACS numbers: $98.80 . \mathrm{Cq}, 14.80 . \mathrm{Bn}$
\end{abstract}

Submitted to: Class. Quantum Grav.

Preprint: RBRC1042

\section{Introduction}

The current results from the Large Hadron Collider (LHC) completed the particle zoo of the Standard Model (SM) of particle physics by the discovery of a Higgs boson [1, 2], while at the same time showing no signs of any beyond the SM physics. The first results from the Planck satellite further confirm the inflationary scenario for the early Universe [3] and favour a simple inflationary scenario with only one slow rolling scalar field. The question remains, whether it is needed to add more particle states to the SM to make the inflation possible, or can it be explained with the already present ones?

Usual considerations require for the potential of the scalar field, responsible for inflation, to be extremely flat. The initial density perturbations can be generated with the observed amplitude by a chaotic inflation with a scalar field with the quartic self-coupling $\lambda \sim 10^{-13}$ or mass $m \sim 10^{13} \mathrm{GeV}$ [4]. There are no particles in the SM with such properties, but it turns out that there is another option. Modification of the gravitational interactions of a scalar particle

$$
\delta S_{\mathrm{NM}}=\int \mathrm{d}^{4} x \sqrt{-g}\left[-\xi \Phi^{\dagger} \Phi R\right],
$$

can lead to a good slow-roll behaviour for arbitrary large $\lambda$ (at the cost of large coupling $\xi$ ). A field $\Phi$ with a large (order 0.1) quartic self-coupling is present in the SM-the Higgs boson. The inflationary model using the SM Higgs boson with the non-minimal coupling (1) will be called Higgs inflation (HI).

The first step in the analysis of the inflation with the non-minimal coupling is the classical solution of the slow-roll evolution. It can be simplified (as far as the equations of motion for 
the action with the addition of (1) look rather complicated [5]) by a suitable change of the dynamical variables, which removes the non-minimal coupling at the cost of the modification of the scalar potential (and interactions with other particles of the theory). This variable change is traditionally called 'conformal transformation', and the theory in the new variables is referred to as the theory in the 'Einstein frame' (as opposed to the original 'Jordan frame'). After this change all the formulas for the standard single-field inflationary slow roll, and generation of the density perturbations can be used, with the result being in extraordinary good agreement with the Planck observations. The details of the full model are described in section 2.

The inflationary scenario in HI depends on the absence of additional new physics above the electroweak scale, but of course variations of the setup are possible. Some examples are described in section 7. All other experimental observations, required beyond the SM physics, namely neutrino oscillations, dark matter and baryon asymmetry of the Universe can be explained with the extensions of the SM that do not affect its properties above the electroweak scale. One possibility is an extension of the SM with right-handed (sterile) neutrinos, for example, $\nu$ MSM [6-8].

A significantly more complicated analysis is required beyond the tree-level approximation. With $\xi \gg 1$, as required by the $\mathrm{CMB}$ observations, the operator (1) introduces new scales in the model below the Planck mass: $M_{P} / \xi$ and $M_{P} / \sqrt{\xi}$, and the model requires some form of the high-energy (UV) completion. While the tree-level calculations of the inflation and generation of the primordial density perturbations are performed at lower values of momenta, the predictions of the model with the quantum corrections depend on the completion. First, the standard approach of perturbation theory, well formulated for a theory above a trivial vacuum, cannot be applied, and even in the analysis of perturbations on top of a classical background (which is relevant for inflation), it is impossible to move beyond the lowest order tree-level calculations without additional assumptions. Various assumptions about the completion of the theory up to the high momentum scales allow us to make different levels of predictions. The weaker one, scale invariance for the theory in UV, allows us to work within a form of effective theory during inflation. A stronger set of assumptions, namely the absence of the quadratic divergences, together with a definite prescription for the subtraction rules for the divergent diagrams, allows us to relate the low-energy and inflationary sectors of the theory. The role of the various scales is discussed in section 4, while the calculation of the quantum corrections is outlined in section 5.

As far as there are no additional scales in the model between the electroweak and inflationary scales, a bound can be made on the Higgs mass. If the Higgs mass is low, the effective Higgs potential may become negative at high scales. If this happens below the inflationary scale $M_{P} / \sqrt{\xi}$, the inflation will end up in this new minimum of the potential, instead of the electroweak phase. An even more stringent bound may exist from the modification of the inflationary potential by loop corrections, but this bound depends on the details of the quantization of the theory. The recent LHC results gave the Higgs mass (within the experimental and theoretical errors) at the boundary value (see section 6).

\section{Non-minimal coupling to gravity and inflation}

\subsection{Inflationary scalar sector and inflation}

One of the simplest inflationary setups is the chaotic inflation [4]. In this approach, the field starts at a large value and rolls slowly towards its origin. The slow roll is obtained due to the 'Hubble friction' connected with the expansion of the universe. Then, if one requires 
the density perturbations of the field, generated during inflation, to have the observed value, a significant constraint on the potential is obtained. For the quartic potential this means, in particular, that the coupling constant is of the order of $\lambda \sim 10^{-13}$. Such particle is not present in the SM, and it is not easy to have this particle coupled to the SM, as far as for not very small couplings they would induce radiative corrections, spoiling the flatness of the potential. However, there is a way out of this unfortunate situation. Let us note that a scalar field in general may be coupled non-minimally to gravity, i.e. in addition to the trace of the energymomentum tensor gravity may 'feel' the scalar field background. This type of coupling is even inevitable, as renormalization of a scalar field in the curved spacetime requires introduction of divergent counter-terms of this form [9]. The action of the scalar field with this term is

$$
S_{J}=\int \mathrm{d}^{4} x \sqrt{-g}\left[-\frac{M^{2}}{2} R-\xi \frac{h^{2}}{2} R+\frac{\partial_{\mu} h \partial^{\mu} h}{2}-V(h)\right],
$$

where $h$ denotes the scalar field, and the potential for the case of the Higgs field is

$$
V(h)=\frac{\lambda}{4}\left(h^{2}-v^{2}\right)^{2},
$$

with the vacuum expectation value of the field $v$. The metric signature used is $(+,-,-,-)$, and the special value, corresponding to the conformal coupling, is $\xi=-1 / 6$. Note that in the case of the Higgs field $h$ corresponds only to the radial mode, $\Phi^{\dagger} \Phi=h^{2} / 2$, or in the unitary gauge, $\Phi=\frac{1}{\sqrt{2}}\left(\begin{array}{l}0 \\ h\end{array}\right)$. The effects from the angular modes and interactions with other particles will be discussed later in sections 2.2 and 5 . The first two terms lead to an effective change of the Planck mass in the presence of the scalar background $M_{P, \text { eff }}^{2} \sim M^{2}+\xi h^{2}$.

It is rather cumbersome to analyse inflation directly with the action (2) (in the 'Jordan frame'). The equations of motion are entangled between the field and the metric (see e.g. [5]), the quadratic part of the action for the small perturbations contains kinetic mixing between the perturbations of the scalar field and the trace of the metric. The simplest way to work with this action is to get rid of the non-minimal coupling to gravity by a suitable change of variables, which is conventionally called conformal transformation from the Jordan frame to the Einstein frame $\dagger$ (see, e.g., [5, 13]):

$$
g_{\mu \nu} \rightarrow \hat{g}_{\mu \nu}=\Omega^{2} g_{\mu \nu}, \quad \Omega^{2}=\frac{M^{2}+\xi h^{2}}{M_{P}^{2}},
$$

where $M_{P} \equiv 1 / \sqrt{8 \pi G_{N}}=2.44 \times 10^{18} \mathrm{GeV}$ is the reduced Planck mass. The parameters $M$ and $M_{P}$ differ for the non-zero vacuum expectation value of $\langle h\rangle=v$, but for most of the situations, we will have $\xi v \ll M$ and set $M \simeq M_{P}$ (with exceptions of sections 3.1.3 and 7.2 , where the vacuum contribution of the non-minimally coupled term actually dominates the Planck mass). This transformation leads to a non-minimal kinetic term for the Higgs field. It is also convenient to replace $h$ with a new canonically normalized scalar field $\chi$, satisfying the relation

$$
\frac{\mathrm{d} \chi}{\mathrm{d} h}=\sqrt{\frac{\Omega^{2}+\frac{3}{2} M_{P}^{2}\left(\Omega^{2}\right)^{\prime 2}}{\Omega^{4}}}=\sqrt{\frac{1+\left(\xi+6 \xi^{2}\right) h^{2} / M_{P}^{2}}{\left(1+\xi h^{2} / M_{P}^{2}\right)^{2}}},
$$

where' means the derivative over $h$. Note that this redefinition is only possible for one real scalar field, and the generic situation with several scalar fields leads to the complicated

$\dagger$ The equivalence of the analysis at the tree level in both the Jordan and Einstein frames was studied by several authors [10-12]. Actually, at the tree level the comparison is effectively reduced to the observation that with the oneto-one correspondence of the classical solutions in any variables, the perturbations on top of it in different frames (or, more precisely, different variable choices) are connected by a linear transformation and are equivalent. At higher orders, the situation is more involved due to non-renormalizability of the theory, see section 5. 
structure of the kinetic terms (see discussion in section 2.2 and [14]). Finally, the action in the Einstein frame is

$$
S_{E}=\int \mathrm{d}^{4} x \sqrt{-\hat{g}}\left[-\frac{M_{P}^{2}}{2} \hat{R}+\frac{\partial_{\mu} \chi \partial^{\mu} \chi}{2}-U(\chi)\right],
$$

where $\hat{R}$ is calculated using the metric $\hat{g}_{\mu \nu}$ and the potential is rescaled with the conformal factor

$$
U(\chi)=\frac{1}{\Omega^{4}[h(\chi)]} \frac{\lambda}{4}\left[h^{2}(\chi)-v^{2}\right]^{2} .
$$

We will, rather ambiguously, write the potential $U$ and the scale factor $\Omega$ as the functions of either $h$ or $\chi$, which should not lead to problems, as far as $h$ and $\chi$ can be expressed one through another in a unique way with the solution of (5).

The analysis of the inflation in the Einstein frame is now straightforward and follows the usual slow-roll approach. The slow-roll parameters (in notations of [15]) are easy to express analytically as the functions of the field $h$ using (5) and (7)

$$
\begin{aligned}
& \epsilon=\frac{M_{P}^{2}}{2}\left(\frac{\mathrm{d} U / \mathrm{d} \chi}{U}\right)^{2}=\frac{M_{P}^{2}}{2}\left(\frac{U^{\prime}}{U} \frac{1}{\chi^{\prime}}\right)^{2}, \\
& \eta=M_{P}^{2} \frac{\mathrm{d}^{2} U / \mathrm{d} \chi^{2}}{U}=M_{P}^{2} \frac{U^{\prime} \chi^{\prime}-U^{\prime} \chi^{\prime}}{U \chi^{\prime 3}},
\end{aligned}
$$

with ' meaning derivative over $h$. Slow-roll ends at $\epsilon \simeq 1$, with the corresponding field value $h_{\text {end }}$. The observed modes left the horizon when the field value equals $h_{N}$ which is determined by the number of inflationary $e$-foldings in the Einstein frame,

$$
N=\int_{h_{\text {end }}}^{h_{N}} \frac{U \chi^{\prime 2}}{U^{\prime}} \frac{\mathrm{d} h}{M_{P}^{2}} \simeq \frac{3}{4}\left[\left(\xi+\frac{1}{6}\right) \frac{h_{N}^{2}-h_{\text {end }}^{2}}{M_{P}^{2}}-\ln \frac{1+\frac{\xi h_{N}^{2}}{M_{P}^{2}}}{1+\frac{\xi h_{\text {end }}^{2}}{M_{P}^{2}}}\right],
$$

where we neglected the quadratic part of the potential. Note that in the Jordan frame the additional ratio of conformal factors enters in the $e$-folding number leading to another definition [16]. The $N$ itself is determined from the scale of the observed mode and evolution of the Universe after the inflation, see section 3.2. To generate the proper amplitude of the density perturbations, the potential should satisfy at $h_{N}$ the normalization condition

$$
\frac{U}{\epsilon}=24 \pi^{2} \Delta_{\mathcal{R}}^{2} M_{P}^{4} \simeq\left(0.0276 M_{P}\right)^{4} .
$$

With the non-minimal coupling as an additional parameter this condition becomes a relation between $\xi$ and $\lambda$, instead of a condition on just $\lambda$. The inflationary predictions (see, e.g., [15]) for the CMB spectrum parameters are then given by the expressions for the spectral index $n_{s}$ and the tensor-to-scalar perturbation ratio $r$,

$$
n_{s}=1-6 \epsilon+2 \eta, \quad r=16 \epsilon,
$$

calculated at $h_{N}$.

Let us immediately note a couple of the features of the obtained action. First, if the Jordan frame potential $V(h)$ is quartic, then, for high values of the field $\xi h^{2} \gg M_{P}^{2}$, the resulting Einstein frame potential $U$ is flat. This suggests that the resulting theory provides a 'better' inflation than the minimally coupled one. See [10, 13, 17] for the discussion of other power-law potentials and non-minimal couplings. 


\subsection{Interaction with other particles}

Let us describe the interaction of the scalar with other particles, neglected in the previous section. First, note that the Higgs boson itself is a complex doublet $\Phi$. The generalization of the conformal transformation (4) is rather obvious: using $\Omega^{2}=1+2 \xi \Phi^{\dagger} \Phi / M_{P}^{2}$ as the conformal factor, we obtain the Einstein frame action as

$S_{\mathrm{E}}=\int \mathrm{d}^{4} x \sqrt{-g}\left[-\frac{M_{P}}{2} \hat{R}+\frac{3 M_{P}^{2}}{4} \frac{\partial_{\mu} \Omega^{2}}{\Omega^{2}} \frac{\partial^{\mu} \Omega^{2}}{\Omega^{2}}+\frac{D_{\mu} \Phi^{\dagger} D^{\mu} \Phi}{\Omega^{2}}-\frac{V\left(\Phi^{\dagger} \Phi\right)}{\Omega^{4}}\right]$,

where $D_{\mu}$ is the gauge covariant derivative. Note that only the kinetic term for the radial mode can be taken into the canonic form by the field redefinition (5), while the derivative interaction of the other components with the radial mode (third term) cannot be removed in general. The gauge boson and fermion kinetic terms are invariant (at least classically) under the conformal transformations (4) augmented by the field transformations

$$
A_{\mu} \rightarrow A_{\mu}, \quad \psi \rightarrow \hat{\psi}=\Omega^{-3 / 2} \psi .
$$

The only terms that are changed are the massive terms. For the gauge bosons, this is due to the rescaling of the third term in (13), and for the fermions, the Yukawa terms change to

$$
S_{E, \text { Yukawa }}=\int \mathrm{d}^{4} x \sqrt{-\hat{g}}\left[y \bar{\psi}_{R} \frac{\Phi^{\dagger}}{\Omega} \psi_{L}+\cdots\right] .
$$

In the unitary gauge $\Phi=\frac{1}{\sqrt{2}}\left(\begin{array}{l}0 \\ h\end{array}\right)$, these changes mean the change $h \rightarrow h(\chi) / \Omega(\chi)$ in all the gauge boson and fermion mass terms.

As in the potential, the powers of $h$ and $\Omega$ are the same in the numerator and denominator, so that the Einstein canonically normalized field $\chi$ always enters the action in the same combination. Specifically, during inflation (at high values of the field), the radial scalar mode decouples from the other fields, while leaving all particle masses proportional to $M_{P} / \sqrt{\xi}$.

If there are additional scalars, the modifications also involve the modifications in kinetic terms, analogously to the third term in (13) for a minimally coupled scalar (see e.g. $[14,18,19])$.

A convenient description of the SM in the Einstein frame can be obtained by the definition $\Phi=\exp \left[2 i \pi(x)^{a} T^{a}\right]\left(\begin{array}{c}0 \\ h(x) / \sqrt{2}\end{array}\right)$, with the $\mathrm{SU}(2)$ generators $T^{a}=\tau^{a} / 2$. Then the action for the scalar mode $h(\chi)$ is $(6)$, and the rest corresponds to the chiral electroweak action with the 'vacuum expectation value' set to $h(\chi) / \Omega$, see [20,21].

\section{Predictions for cosmology, universe history}

\subsection{Inflationary stage}

There are several interesting limiting situations, depending on the choice of the parameters in the potential and gravitational sector [5, 22].

3.1.1. Large $\xi$ limit (Higgs inflation) The HI case corresponds to the large $\xi \gg 1$ and negligible vacuum contribution of the non-minimal coupling to the Planck mass, $\xi v \ll M_{P}$. Then equation (5) can be solved approximately as

$$
\chi \simeq \begin{cases}h & \text { for } h \ll \frac{M_{P}}{\xi}, \\ \sqrt{\frac{3}{2}} M_{P} \ln \Omega^{2}(h) & \text { for } h \gg \frac{M_{P}}{\xi} .\end{cases}
$$



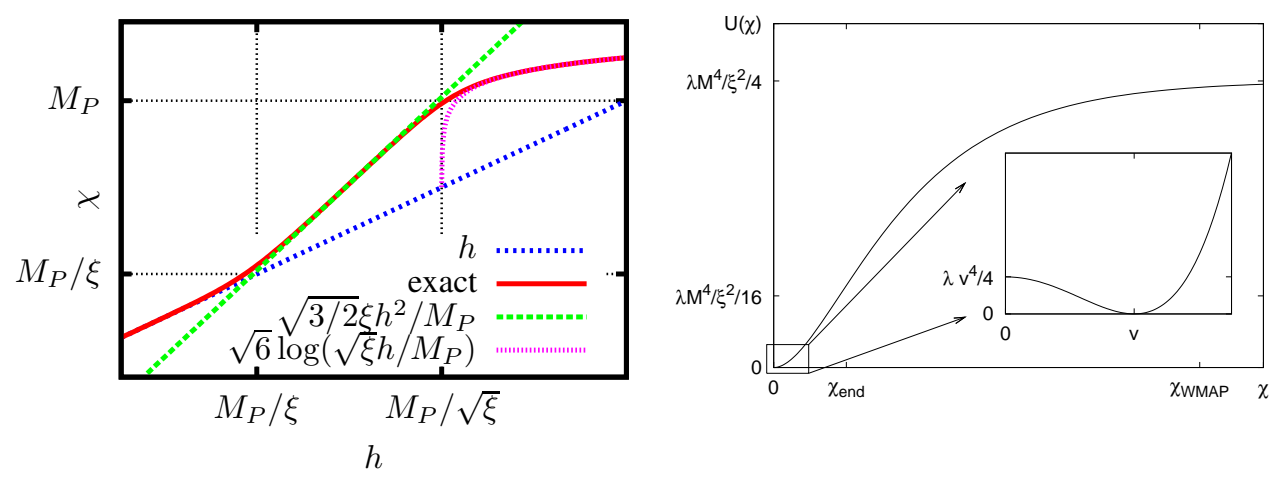

Figure 1. Left: dependence of $\chi$ (the Einstein frame Higgs field) on $h$ (the Jordan frame Higgs field), logarithmic scale. Right: effective potential in the Einstein frame. The insert magnification is not to scale.

This relation is illustrated in figure 1 . Using this relation, we can explicitly write the potential for the large Higgs background $h, \chi \gg M_{P} / \xi$ as

$$
U(\chi) \simeq \frac{\lambda M_{P}^{4}}{4 \xi^{2}}\left(1-\mathrm{e}^{-\frac{2 \chi}{\sqrt{6} M_{P}}}\right)^{2} .
$$

For a small field $h, \chi \ll M_{P} / \xi$, the potential turns into the usual SM quartic potential with a negative mass. Figure 1 schematically shows the potential.

Note that there are actually two interesting energy scales for $\xi \gg 1$. The lowest one is $M_{P} / \xi$, corresponding to the scale where deviation from the SM becomes significant. The higher one is $M_{P} / \sqrt{\xi}$, which is the energy scale at inflation and typical particle scale for excitation masses during inflation. The Hubble scale (in the Einstein frame) during inflation is $H^{2} \simeq \lambda M_{P}^{2} /\left(12 \xi^{2}\right)$.

The potential (17) is exponentially flat for large field values and provides the slow-roll inflation. From (8), (9) and (3), we obtain

$$
\epsilon \simeq \frac{4 M_{P}^{4}}{3 \xi^{2} h^{4}}, \quad \eta \simeq \frac{4 M_{P}^{4}}{3 \xi^{2} h^{4}}\left(1-\frac{\xi h^{2}}{M_{P}^{2}}\right) .
$$

Inflation ends at $h_{\text {end }} \simeq(4 / 3)^{1 / 4} M_{P} / \sqrt{\xi} \simeq 1.07 M_{P} / \sqrt{\xi}$ (and $\left.\chi_{\text {end }} \simeq 0.94 M_{P}\right)$. Using (10), we obtain the field value corresponding to the horizon crossing of the observed CMB modes $h_{N} \simeq 9.14 M_{P} / \sqrt{\xi}$. Thus, the CMB normalization (11) requires

$$
\xi \simeq 47000 \sqrt{\lambda},
$$

where $\lambda$ is the Higgs boson self-coupling constant, taken at an inflationary scale.

Finally, to the lowest order in $1 / \xi$, the spectral index and the tensor-to-scalar perturbation ratio are

$$
n_{s} \simeq 1-8 \frac{4 N+9}{(4 N+3)^{2}} \simeq 0.967, \quad r \simeq \frac{192}{(4 N+3)^{2}} \simeq 0.0031,
$$

where the number of $e$-foldings $N \simeq 57.7$ (see section 3.2). Note that there are higher corrections suppressed by $1 / \xi$ and $1 / N$ to these formulae. See figure 2 for the good agreement of this result with the latest measurements. The inflation model in the Einstein frame is a simple one field slow-rolling inflation, with all extra degrees of freedom much heavier than the Hubble scale $\left(m \sim M_{P} / \sqrt{\xi} \gg H \sim M_{P} / \xi\right)$, so it does not predict any significant non-Gaussianities in the spectrum. 


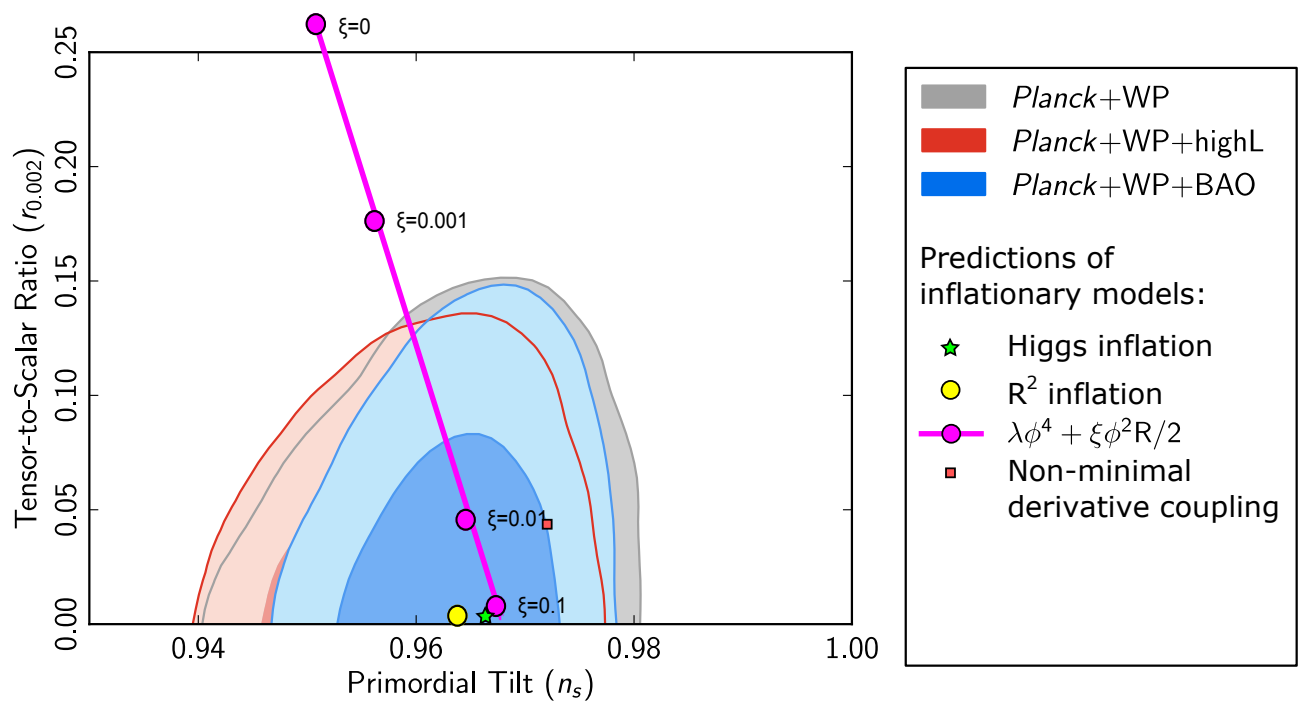

Figure 2. Predictions (at a tree level, which coincides with the radiatively corrected results in the scale-invariant quantization, choice I of section 6) from the inflationary models [23-25] and the Planck satellite [3] observed bounds.

3.1.2. Nearly quartic inflation Let us note that the case of small $\xi$, though not relevant for $\mathrm{HI}$, provides a variation of the usual quartic potential inflation which is in agreement with the recent experiments. The analysis for this case can be performed by approximating (10) as (which by coincidence works quite well for all values of $\xi$ )

$$
(1+6 \xi) \frac{h_{N}^{2}}{M_{P}^{2}} \simeq 8(N+1) .
$$

Plugging this into (8), (9) and (12), the CMB predictions are obtained, see [25] for details. The result smoothly interpolates with the growing $\xi$ between the minimally coupled (now strongly disfavored by Planck) and HI points. For $\xi \gtrsim 0.003$ the predictions enter into the $2 \sigma$ experimentally allowed region, without introducing any new scales of the type discussed in sections 4 and 5 . The required quartic coupling, however, is still very small, nearly unchanged from the pure quartic value $\lambda \simeq 1.5 \times 10^{-13}$, so a dedicated inflaton field is required.

3.1.3. Induced gravity Another interesting case emerges when the Planck mass is generated by the vacuum expectation value of the scalar field via the non-minimal coupling term, $M_{P}^{2}=\xi v^{2}$. This corresponds to the very early works about the non-minimal coupling [22, 26-32]. With the non-minimal coupling given by (19), the value for $v=M_{P} / \sqrt{\xi}$ is relatively close to the GUT scale for $\lambda \sim 1$. This suggests that the identification of the inflaton field may be made with the GUT scale Higgs boson. The conformal factor is in this case

$$
\Omega^{2}=\frac{\xi h^{2}}{M_{P}^{2}} \equiv \frac{h^{2}}{v^{2}} .
$$

The relation between the Jordan and Einstein frame fields is obtained in an exact form from (5) and (22) as

$$
h=v \exp \left(\frac{\chi}{\sqrt{6+1 / \xi} M_{P}}\right),
$$


The Einstein frame potential is now equal to (17) for all values of the field. However, the field $\chi$ becomes exactly decoupled from all the gauge (and fermion) fields it was giving mass to. As described in section 2.2 all the masses of the gauge bosons and fermions, associated with the Higgs boson, are proportional to $h / \Omega$. With the conformal factor $(22)$, this is constant $M_{P} / \sqrt{\xi}$. Thus, the scalar field completely decouples from the matter fields and couples to them only via gravity. Note, however, that it is coupled to all other non-conformal terms in the actions, specifically to the other scalar particles (e.g. the SM Higgs boson) via the conformal factor. These interactions are suppressed by the Planck mass in the exponent of (23).

A singlet scalar of this type is an exact equivalent of the scalaron which appears after the conformal transformation of $R^{2}$ gravity [33], and its inflationary phenomenology was described recently in detail in [24, 34-36].

\subsection{Post inflationary cosmology}

Another notable feature of the HI model is that the evolution of the Universe after inflation, i.e. a preheating mechanism is fixed. This removes the ambiguity in the CMB parameter definition associated with the unknown number of $e$-foldings of inflation, which is often indicated on the plots with inflationary predictions.

The preheating in the model was analysed in [37,38]. After the end of inflation, $\chi<M_{P}$ ( $h<M_{P} / \sqrt{\xi}$ ), the potential (17) can be approximated by a quadratic function (with just a small quartic part around the origin corresponding to the SM potential for $\left.\chi<M_{P} / \xi\right)$. The field $\chi$ starts to oscillate and the expansion of the universe corresponds to the matter dominated regime. During this regime the SM particles (mostly $W$ and $Z$ bosons) are produced at the moments when the Higgs field crosses zero. At first, they decay faster than the oscillations of the field $\chi$, not leading to effective preheating (transfer of energy from the inflaton to the relativistic SM degrees of freedom). At the moment when they start to live long enough the parametric resonance starts, the concentration of the gauge boson rises and they start to effectively annihilate into light SM particles [37], leading to nearly immediate preheating. Another significant reheating mechanism is the production of relativistic excitations of the Higgs field itself, which leads to the lower bound on the reheating temperature. The preheating temperature is estimated as $T_{r} \sim 0.3-1.1 \times 10^{14} \mathrm{GeV}$. Numerically more precise analysis of [38] leads to similar results for the onset of parametric resonance. There are a number of effects which are not yet carefully taken into account in the reheating analysis (Pauly blocking of the produced fermions, the decay of the gauge bosons at the moments of the zero crossing, when the particle description fails, back reaction effects, etc), so the value of the reheating temperature may still differ a bit from the given values.

Note that although determining the temperature when the whole matter in the universe is properly thermalized may be a hard problem, it is easy to estimate the absolute lower bound on the effective temperature when the expansion of the universe switches to the radiation dominated regime (which is the moment relevant for the calculation of the number of $e$ foldings in the next paragraph). For this, let us note that at $h \lesssim M_{P} / \xi$ the Higgs potential switches to the quartic one, meaning that the expansion is effectively radiation dominated. Equating the potential to the thermal energy density at this moment one obtains the estimate of

$$
T_{r} \gtrsim\left(\frac{15}{2 \pi^{2} g_{*} \lambda}\right)^{1 / 4} \frac{M_{p}}{47000} \gtrsim 10^{13} \mathrm{GeV},
$$

so further calculation of the reheating is not going to change the $e$-folding number beyond the error quoted in (25). Note that this bound moves up for smaller Higgs masses. 
The number of $e$-foldings in the Einstein frame (where the standard sigle-field inflationary analysis can be used) between the modes corresponding to the present day pivot scale $k / a_{0}$ exiting the horizon and the end of inflation is

$$
\begin{aligned}
N & =\ln \left(\frac{\sqrt{\pi}}{270^{1 / 4}} \frac{\tilde{g}_{0}^{1 / 3}}{g_{* r}^{1 / 12}}\right)-\ln \frac{k}{a_{0} T_{0}}-\ln \frac{M_{P}}{U_{N}^{1 / 4}} \frac{U_{e}^{1 / 4}}{U_{N}^{1 / 4}}-\frac{1}{3} \ln \frac{U_{e}^{1 / 4}}{\rho_{r}^{1 / 4}} \\
& =58.9-\frac{1}{3} \ln \frac{U_{e}^{1 / 4}}{\rho_{r}^{1 / 4}} \simeq 57.7 \pm 0.2,
\end{aligned}
$$

where $k / a_{0}=0.002 \mathrm{Mpc}$ is the scale of the observed perturbations used for the normalization of the spectrum, $g_{* r}=106.75$ and $\tilde{g}_{0}=43 / 11$ are the effective number of d.o.f. at reheating and now, $T_{0}=2.725 \mathrm{~K}$ is the CMB temperature, $U_{N}=U\left(h_{N}\right)$ and $U_{e}=U\left(h_{\text {end }}\right)$ are the Einstein potentials at the moment of horizon crossing and at the end of slow roll and $\rho_{r}$ is the thermal energy density at preheating. The error here comes from the reheating estimates in [37] and may be somewhat underestimated.

Knowing the exact value of $N$ allows for the precise prediction of the spectral index and tensor-to-scalar ratio (20). In fact, this precise prediction even allows us to distinguish between the $\mathrm{HI}$ and the $R^{2}$ inflation, both of which have similar inflationary potentials, but different preheating mechanisms $[24,36]$.

\section{Naturalness and strong coupling—-tree-level analysis}

Let us find the scale associated with the non-minimal coupling (1). To do this we expand the metric on top of the flat background $\eta_{\mu \nu}$ (we work here in the Jordan frame, the Einstein frame result is the same, but is slightly more cumbersome to obtain, see [39])

$$
g_{\mu \nu}=\eta_{\mu \nu}+\frac{1}{M_{P}} \gamma_{\mu \nu}
$$

where $\gamma_{\mu \nu}$ has a canonically normalized kinetic term. The leading interaction term from (1) is

$$
\frac{\xi}{M_{P}} h^{2} \eta^{\mu \nu} \partial^{2} \gamma_{\mu \nu}
$$

On dimensional grounds this term makes the tree-level scattering amplitude grow at high energies, and eventually violates the unitarity bound [40] at the energies of the order of $E \sim M_{P} / \xi$ [41-45] (there are some cancellations for the case of a real field $h$ without a potential, which are no longer true for complex or interacting scalar fields [44]). This scale is low; for example, it is much smaller than the energy density scale at inflation $\lambda^{1 / 4} M_{P} / \sqrt{\xi}$ or inflationary field values $h \sim M_{P} / \sqrt{\xi}$. It is still slightly (parametrically in $\sqrt{\lambda}$ ) larger than the inflationary Hubble scale (which is the relevant momentum scale for processes during inflation) $H \sim \lambda^{1 / 2} M_{P} / \xi$, but this is quite a tight call.

Following the standard rules of quantum field theory, this observation is very dangerous for the Higgs inflation. Actually, usual assumptions of the effective field theories suggest that if there is an energy scale $\Lambda \sim M_{P} / \xi$ in the theory, corresponding to the violation of the tree-level unitarity, then some new physics enters at this scale, and leads to the appearance of the operators $h^{(4+n)} / \Lambda^{n}$ in the action, with some unknown coefficients. It is then usually assumed that all these coefficients are some uncorrelated numbers of order 1 . Thus, the analysis of the theory for $h>\Lambda \sim M_{P} / \xi$ is assumed to be impossible, rendering the inflationary analysis of the previous section (with $h \sim M_{P} / \sqrt{\xi} \gg M_{P} / \xi$ ) false. However, it is still possible to obtain useful predictions from the HI, if one uses a more generic 
understanding of effective field theories. First, we will check that tree-level calculations are safe if the classical background is taken into account (instead of the vacuum one). Then, we will formulate assumptions about the theory that make the possible calculation of the quantum corrections. Weaker assumptions allow us to work in a form of an effective theory in an inflationary regime and the low-energy regime separately, and stronger assumptions make the connection between these regimes possible.

Let us first study whether our lowest order approximation for the universe evolution can be trusted (i.e. the inflation itself and the generation of density perturbations proceed as described). The processes we study during inflationary expansion occur around the classical background, that is, the slow-roll solution during the inflation, so the (quantum) analysis should be made for the perturbations $\delta \Theta(x)$ on top of the classical background $\Theta_{0}[39,46]$ :

$$
\Theta(x)=\Theta_{0}+\delta \Theta(x),
$$

where $\Theta$ stands for all the fields of the model. To estimate this cut-off scale $\Lambda(\Theta)$, we should substitute (28) into the action and look for the expansion in powers of $\delta \Theta$ :

$$
\frac{\delta \Theta^{n}}{\Lambda_{N}\left(\Theta_{0}\right)^{n-4}},
$$

and take the minimal scale $\Lambda\left(\Theta_{0}\right)=\min _{N} \Lambda_{N}\left(\Theta_{0}\right)$ (this rounds off some corners, like possible cancellations or amplitude behaviour at large $N$, which are irrelevant for the current discussion). So, instead of the same cut-off for the momentum and for the values of the field, we have the energy scale depending on field background, background-dependentcut-off scale, below which the tree-level calculation is possibleł. Note that even with this definition the theory is not complete [39] and enters into a strongly coupled regime above the energy $\Lambda\left(\Theta_{0}\right)$, where it is unitarized by some yet unknown mechanism (own strong dynamics, asymptotic safety or even new perturbative physics), cf [45, 47].

The proposed approach can be carried both in the Jordan and Einstein frames [39], bearing in mind that the cut-off scales are connected by the conformal scaling

$$
\Lambda_{J}=\Omega \Lambda_{E} \text {. }
$$

Here, we will review only the Jordan frame estimate; see [39] for details and the calculation in the Einstein frame. For the scalar-gravity sector, we have the expansion

$$
g_{\mu \nu}=\bar{g}_{\mu \nu}+\frac{1}{M_{P}} \gamma_{\mu \nu}, \quad h=\bar{h}+\delta h .
$$

The quadratic Lagrangian for the excitations has the form

$$
\begin{aligned}
\mathcal{L}^{(2)}= & -\frac{M_{P}^{2}+\xi \bar{h}^{2}}{8 M_{P}^{2}}\left(\gamma^{\mu \nu} \square \gamma_{\mu \nu}+2 \partial_{\nu} \gamma^{\mu \nu} \partial^{\rho} \gamma_{\mu \rho}-2 \partial_{\nu} \gamma^{\mu \nu} \partial_{\mu} \gamma-\gamma \square \gamma\right) \\
& +\frac{1}{2}\left(\partial_{\mu} \delta h\right)^{2}+\xi \bar{h}\left(\square \gamma-\partial_{\lambda} \partial_{\rho} \gamma^{\lambda \rho}\right) \delta h,
\end{aligned}
$$

where $\gamma=\gamma_{\mu}^{\mu}$. We retained here only the terms with two derivatives of the excitations as they determine the UV behaviour of the scattering amplitudes and hence the unitarity violation scale. Note that in the non-trivial background, a large kinetic mixing between the trace of the metric and the scalar perturbations is present [48-51]. The change of variables

$$
\begin{aligned}
\delta h & =\sqrt{\frac{M_{P}^{2}+\xi \bar{h}^{2}}{M_{P}^{2}+\left(\xi+6 \xi^{2}\right) \bar{h}^{2}}} \delta \hat{h}, \\
\gamma_{\mu \nu} & =\frac{M_{P}}{\sqrt{M_{P}^{2}+\xi \bar{h}^{2}}} \hat{\gamma}_{\mu \nu}-\frac{2 \xi \bar{h} \bar{g}_{\mu \nu}}{\sqrt{\left(M_{P}^{2}+\xi \bar{h}^{2}\right)\left(M_{P}^{2}+\left(\xi+6 \xi^{2}\right) \bar{h}^{2}\right)}} \delta \hat{h}
\end{aligned}
$$

$\ddagger$ We often use the widely spread term 'cut-off', while strictly speaking we always mean 'the momentum scale of the violation of the tree-level unitarity'. 

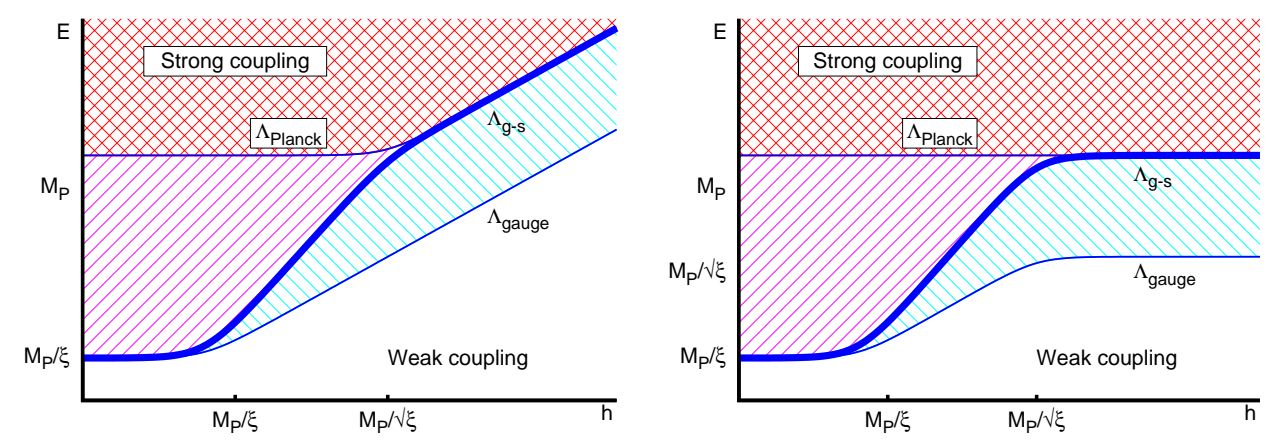

Figure 3. Schematic depiction of the cut-offs (scales of violation of the tree-level unitarity) in the Jordan (left) and Einstein (right) frames for different sectors of the theory. $\Lambda_{\mathrm{g}-\mathrm{s}}$ corresponds to the inflaton (scalar sector) scatterings, $\Lambda_{\text {gauge }}$ to the gauge boson scattering and $\Lambda_{\text {Planck }}$ is the graviton scattering.

diagonalizes the kinetic term. The unitarity violation scale can be read out of the operators with a dimension higher than 4 . The leading operator is the cubic scalar-graviton interaction $\xi(\delta h)^{2} \square \gamma$, which is written in terms of the canonical fields $\delta \hat{h}, \hat{\gamma}_{\mu \nu}$ obtains the dimensional coefficient corresponding to the cut-off scale

$$
\Lambda_{\mathrm{g}-\mathrm{s}}^{J}(\bar{h}) \sim \frac{M_{P}^{2}+\left(\xi+6 \xi^{2}\right) \bar{h}^{2}}{\xi \sqrt{M_{P}^{2}+\xi \bar{h}^{2}}} .
$$

At the low-field background $\bar{h} \ll M_{P} / \xi$, this formula readily reproduces the $M_{P} / \xi$ cut-off of the works [41-44]. It is smaller than the Planck mass, but for $\xi \sim 10^{4}$, it is still way above the reach of the collider experiments.

At large inflationary fields, $\bar{h} \gg M_{P} / \sqrt{\xi}$, the cut-off grows linearly with $\bar{h}$ (or reaches Planck scale in the Einstein frame), making the calculation of the inflationary perturbations safe. Note that this coincides with the cut-off in the gravitational (spin-2) sector. The latter is given by the effective Planck mass defined as the coefficient in front of the $R$ term in the Lagrangian, $\Lambda_{\text {Planck }}^{J}=\sqrt{M_{P}^{2}+\xi \bar{\phi}^{2}}$.

In an analogous way one can estimate the cut-off in the gauge sector (see [52] for the detailed calculation in a closely related Higgs-Dilaton model)

$$
\Lambda_{\text {gauge }}^{J}(\bar{h}) \sim \frac{\sqrt{M_{P}^{2}+\xi(1+6 \xi) h^{2}}}{\sqrt{6} \xi} .
$$

The behaviour of the cut-offs (35) and (36) is illustrated in figure 3 both in the Jordan and Einstein frames.

Thus, we can conclude that at a tree level the situation is safe. At inflation, the tree-level cut-offs (35) and (36) are significantly higher than the typical interesting momenta, which are of the order of the Hubble scale $H \sim M_{P} / \xi$. Currently, (after preheating, $h<M_{P} / \xi$ ), we have small temperatures, $T<M_{P} / \xi$, much below the cut-off. The most subtle situation is achieved during the preheating, especially at its early stages. However, although it makes the careful preheating calculation even more complicated, it is unlikely for us to expect that the final preheating results will be significantly different, and the numerical impact of the preheating on the CMB observations is encoded in the number of $e$-foldings (25) and is logarithmically weak. 
One should note that as far as above some of the scales $\Lambda$ discussed in this section, new physics can be expected, additional higher order operators can be present in the theory, suppressed by this scale. Various effects of these operators were analysed in [37, 53], including the possible generation of the baryon asymmetry and sterile neutrino dark matter.

\section{Quantization and loop corrections}

Let us now move to the analysis of the loop corrections to the Higgs inflation. The question of how to calculate them really does not have a unique answer, as far as the action (2) is non-renormalizable. The non-renormalizability of the action means that, in principle, an infinite number of counter-terms should be fixed to obtain predictions from the theory. Strictly speaking, performing scattering experiments at energies below $M_{P} / \xi$ around the electroweak vacuum background does not allow us to distinguish between different potentials at inflation. In the Einstein frame (for the simplicity of the argument), the arbitrarily precise scattering measurement gives a finite number of coefficients in the power series for the Higgs potential around zero (electroweak vacuum). Altering the remaining infinite number of coefficients, it is possible to imitate an arbitrary potential at high values of the field. This means that additional principles (or assumptions about the UV completion) should be used to make the theory predictable. This is inevitable, as far as, though we are working within the boundaries of allowed momenta, we are interested in large field backgrounds, and that takes us beyond the limits of ordinary effective field theory.

The effective field theory around the electroweak background is easy to describe: it is given by the usual SM with power-law corrections suppressed by the $M_{P} / \xi$ scale. To make more precise predictions one needs to measure more coefficients.

A less ordinary setup is needed to obtain the effective field theory at inflation. The crucial observation is that at large Higgs field values, the theory possesses an approximate symmetry - scale transformations in the Jordan frame, or shifts of the Higgs field $\chi \rightarrow$ $\chi+$ const in the Einstein frame. In the Einstein frame, we have the minimally coupled scalar field theory with the potential of the form

$$
U(\chi)=U_{0}\left(1+\sum_{n=1}^{\infty} u_{n} \mathrm{e}^{-\frac{2 n \chi}{\sqrt{6} M}}\right) .
$$

Splitting the field into the perturbations and smooth background as $\chi=\bar{\chi}+\delta \chi$, one can check [39] that all the divergent terms are proportional to the exponents (vanishing at large $\chi$ ) and that all the divergences can be absorbed into the Lagrangian of the form

$$
\mathcal{L}=f^{(1)}(\chi) \frac{\left(\partial_{\mu} \chi\right)^{2}}{2}-U(\chi)+f^{(2)}(\chi) \frac{\left(\partial^{2} \chi\right)^{2}}{M^{2}}+f^{(3)}(\chi) \frac{(\partial \chi)^{4}}{M^{4}}+\cdots,
$$

with the coefficient functions

$$
f^{(i)}(\chi)=\sum_{n=0}^{\infty} f_{n}^{(i)} \mathrm{e}^{-\frac{2 n \chi}{\sqrt{6} M}} .
$$

Although an infinite number of terms are present in this Lagrangian, inflationary predictions at any chosen precision need only a finite set of terms, as far as the exponents are small during inflation and higher order terms are less relevant.

The same form of the contributions appears from the other SM fields, because they couple to the Einstein frame Higgs field in the combination $\mathrm{e}^{-2 \chi / \sqrt{6} M_{P}}$, see section 2.2. The asymptotic shift invariance of the whole action in the Einstein frame (which originates form 
the scale invariance of the Jordan frame action (2)) makes it possible to develop an effective inflationary theory with self-induced radiative corrections under control (cf [54]).

The additional assumption used here is that no contributions, breaking the shift invariance, appear from the UV completion of the theory. Such corrections do not immediately appear from gravity, as the field $\chi$ is coupled to gravity minimally in the Einstein frame (non-perturbative gravitational effects may spoil the picture, but they are model dependent and can be exponentially suppressed [55]). Scale invariance in the Jordan frame suggests that the use of scale-invariant models may be important to address the problem (see $[52,56])$, but no full scale-invariant UV completion is known at the moment.

Even more difficult is the relation of the physics around EW vacuum and the effective inflationary theory. To see this, let us try to estimate the loop corrections to the potential, supposing that at the tree level an exact potential $\lambda U(\chi)$ is known (we explicitly show the coupling constant here). The expansion on top of the background $\bar{\chi}$ has the form

$$
\lambda U(\bar{\chi}+\delta \chi)=\lambda\left[U(\bar{\chi})+\frac{1}{2} U^{\prime}(\bar{\chi})(\delta \chi)^{2}+\frac{1}{3 !} U^{\prime \prime}(\bar{\chi})(\delta \chi)^{3}+\cdots\right] .
$$

Computing loop corrections in, say, the cut-off regularization scheme one generates the divergences of the form:

$$
\begin{aligned}
& \text { in one loop: } \lambda U^{\prime}(\bar{\chi}) \bar{\Lambda}^{2}, \lambda^{2}\left(U^{\prime}(\bar{\chi})\right)^{2} \ln \bar{\Lambda}, \\
& \text { in two loops: } \lambda U^{(I V)}(\bar{\chi}) \bar{\Lambda}^{4}, \lambda^{2}\left(U^{\prime \prime}\right)^{2} \bar{\Lambda}^{2}, \lambda^{3} U^{(I V)}\left(U^{\prime}\right)^{2}(\ln \bar{\Lambda})^{2},
\end{aligned}
$$

where $\bar{\Lambda}$ is the regularization parameter (not to be confused with the tree-level unitarity violation scale of section 4). The first observation is that the divergences have (generally) a functional form different from the original potential, so they cannot be reabsorbed in the redefinition of the coupling constants. The usual exception to this situation corresponds to the renormalizable theories, when the potential is a power series of low enough order. In a generic case, we obtain an infinite number of terms without any evident hierarchy between them.

The possibility for the inflationary case under study is to assume the complete absence of quadratic divergences. In this case, the higher order corrections are suppressed by higher powers of $\lambda$. Note that the theory still needs further determination. First, there is the usual effective theory-like determination - at each order in $\lambda$ there are divergences from lower order terms, leading to the counter-terms that should be fixed from measurements. Second, it is also possible to add any functional $\chi$ dependence at each order in $\lambda$ directly to the tree-level potential. One simple choice to define a theory is to postulate the tree-level potential (7) and make all calculations in dimensional regularization (thus discarding automatically all powerlaw divergences), and choosing a fixed subtraction scheme (for example, $\overline{\mathrm{MS}}$ ). This logic was implicitly used in all works relating electroweak parameters (the Higgs boson mass) to inflationary observations $[49,50,57]$. Note that one can change the subtraction rule by an order 1 constant without significant impact on the results [39].

\section{Modification of CMB predictions and bounds on the Higgs boson mass}

Using the described approach, one can calculate the loop-corrected potential for the inflation. The recipe described in the end of the previous section means writing a Coleman-Weinberg effective potential (for simplicity, we will work exclusively in the Einstein frame in this section), which has the form $m^{4} \ln m^{2}$ for each mass state where the mass is evaluated in the given background. The contribution from the Higgs boson itself is a bit subtle, as its mass squared $m_{\chi}^{2}(\chi)=U^{\prime}(\chi)$ is negative during inflation; however, this complication is connected with the fact that we are using the effective potential calculation away from its 
minimum, while we should use the time-dependent approach as Schwinger-Keldysh or 'inin' formalism [58,59]. However, the Higgs boson mass at inflation is exponentially small, and the corrections due to its contribution are suppressed by an extra power of $\xi$. Thus, working at the lowest order in $1 / \xi$ we can neglect this contribution. The contributions of the other SM fields are not small compared to the tree-level potential

$\Delta U_{1}=\frac{6 m_{W}^{4}}{64 \pi^{2}}\left(\ln \frac{m_{W}^{2}}{\mu^{2}}-\frac{5}{6}\right)+\frac{3 m_{Z}^{4}}{64 \pi^{2}}\left(\ln \frac{m_{Z}^{2}}{\mu^{2}}-\frac{5}{6}\right)-\frac{3 m_{t}^{4}}{16 \pi^{2}}\left(\ln \frac{m_{t}^{2}}{\mu^{2}}-\frac{3}{2}\right)$.

The $\mu$ dependence here is spurious, and is compensated by the renormalization group (RG) running of the coupling constants. With the RG improvement one can choose $\mu=\mu(\chi)$ in some way to minimize the contribution of the logarithms. The result is the (tree-level) RG-enhanced potential

$$
U_{\mathrm{RGE}}=\frac{\lambda(\mu(\chi)) M_{P}^{4}}{4 \xi^{2}(\mu(\chi))}\left(1-\mathrm{e}^{-\frac{2 \chi}{\sqrt{6} M_{P}}}\right)^{2} .
$$

One can add the corrections from $\Delta U_{1}$, associated with the difference of the particle masses, but they are changing logarithmically slowly compared to the tree-level contribution, and we will skip them in the following discussion (in numerical analysis they are easy to take into account, and do not lead to a significant modification of the inflationary results). The RG dependence in the inflationary region is different from the ordinary SM renormalization flow and can be obtained exactly from the requirement to cancel the $\mu$ dependence in (43), see [20]. The RG equations are known in two cases-either the inflationary case with $h(\chi)>M_{P} / \sqrt{\xi}$, or the SM region $H(\chi)<M_{P} / \xi$ (where it is the usual SM RG running). The two RG flows can be connected at any scale between $M_{P} / \xi$ and $M_{P} / \sqrt{\xi}$, without a significant change of the inflationary potential [20], which makes us expect that the effects between these scales (where detailed analysis is impossible) are weak and can be neglected.

The analysis of the impact on for the inflationary observations was performed in a set of works [20, 48-51, 57]. There is a set of differences in the analysis which deserves a comment. Leaving out the earlier works, I will focus on the latest results in [20,50,51]. There are two significant differences. First is related directly to the arbitrariness of the subtraction procedure used to define the theory, and is inevitable. Instead of using a constant $\mu$ in the dimensional regularization in (43), it is possible to use some arbitrary function of the field $\mu=\hat{\mu} F(\chi)$, where $\hat{\mu}$ is used to improve the potential with the usual RG corresponding to changing momentum, and $F(\chi)$ represents the arbitrariness in the subtraction proceduret. Two choices of the function $F(\chi)$ are usually considered:

\begin{tabular}{lcc}
\hline$\mu^{2} / M_{P}^{2} \propto$ & Einstein frame & Jordan frame \\
\hline Choice I & $F_{I}^{2}=1$ & $F_{I}^{2}=\frac{M_{P}^{2}+\xi h^{2}}{M_{P}^{2}}$ \\
Choice II & $F_{I I}^{2}=\frac{M_{P}^{2}}{M_{P}^{2}+\xi h^{2}}$ & $F_{I I}^{2}=1$ \\
\hline
\end{tabular}

The first one is the usual choice for the Einstein frame. At the same time, it is the choice motivated by the scale-invariant quantization in the Jordan frame, where the scale used for the quantum corrections is proportional to the effective Planck mass in the Higgs field background

$\dagger$ In the textbook formulation of the quantum field theory, the function $F(\chi)$ is set to 1 , because this is the choice for the renormalizable theories. If the subtraction is being made with other goals in mind, for example, to retain the scale invariance of the theory on the quantum level, other choices can be made [56, 60]. As far as the inflationary theory is non-renormalizable from the start, the choice $F=1$ is not obviously the best one. 

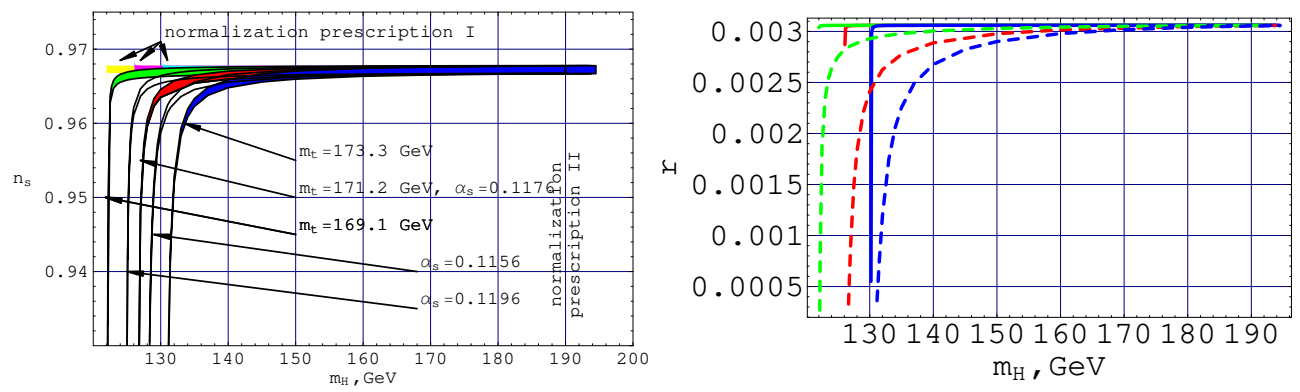

Figure 4. The predictions for the spectral index $n_{s}$ (left) and tensor-to-scalar ratio $r$ (right) with radiative corrections [20]. Note that for the scale-invariant normalization prescription I, the predictions coincide with the tree level, while in the prescription II, the difference becomes more significant at low values of the Higgs mass.

[52]. The second choice is usually explained as the Jordan frame quantization-it corresponds to the constant scale (ordinary quantization rules, breaking the scale invariance at a high Higgs field background) in the Jordan frame, and looks quite peculiar in the Einstein frame. In the studies [20,57], both choices were analysed, while studies [49-51] use choice II only. Using these choices one obtains the RG-enhanced potential (44) taking

$$
\mu_{I}^{2}(\chi)=\frac{y_{t}^{2}}{2} \frac{h(\chi)^{2}}{1+\xi h(\chi)^{2} / M_{P}^{2}}, \quad \mu_{I I}^{2}(\chi)=\frac{y_{t}^{2}}{2} h(\chi)^{2} .
$$

In choice I, the scale $\mu(\chi)$ changes in the same way as the masses of the particles in the limit of high fields (the masses are just constant at $\chi \rightarrow \infty$ in the Jordan frame). Note that the coefficients in front of the logarithms in (43) are all proportional to the tree-level potential (17), it becomes clear that in this choice the overall contribution of the loop corrections only changes the overall normalization of the potential, which leads to the change of the value of $\xi$ in (19), but does not change the shape of the potential and the predictions for the spectral index or tensor-to-scalar ratio. In choice II, the scale $\mu_{I I}(\chi)$ varies significantly during inflation, leading to some changes, most significant at lower Higgs boson masses, see figure 4 . It should be emphasized that choices of the subtraction procedure different from I and II are possible, and it is not clear which is the best one, at least without knowledge of the full UV complete theory, including gravity.

The second difference between [20] and [48, 50,51, 61] is in the coefficient in front of the logarithm (or equivalently to different forms of RG equations at high values of the Higgs field background). The difference is connected with the treatment of the Goldstone modes of the Higgs field. In [20], the calculation is performed in the Einstein frame in the unitary gauge, where the Goldstone modes are explicitly massless, and their contribution to the effective potential is zero. The studies $[48,50,51]$ perform calculations in the Jordan frame, and directly diagonalize the quadratic action for the excitations on top of the background in it, using the linear representation of the Higgs field. In this frame, the question of the masses of the Goldstone modes is more subtle, as far as the evolution of the background field should be taken into account. In short, in the calculation of [20] the masses of the Goldstone modes are exponentially suppressed (in a way similar to the self-contribution of the radial mode driving inflation), and in $[48,50,51])$ they have the mass of the order of gauge and fermion masses. Taking them into account modifies the potential and leads (in prescription II) to different predictions for the cosmological observables, cf prescription II lines in figure 4 (figure 7 in [20]) and figure 3 in [51]. Note in passing that the prescription I leads to the CMB predictions 
coinciding with the tree level (12). The role of Goldstone bosons for the effective potential in the case of an evolving background was further analysed in the studies [59, 62], but only in the context of a minimally coupled theory. However, it can be expected that the presence of the non-minimal coupling term suppresses the Goldstone mode contribution and leads to the result of [20].

As far as the inflationary potential is modified, the predictions for the CMB observables are also changed, figure 4 [20]. In the scale-invariant prescription I, the arguments of the logarithms in the loop contributions to the effective potential (43) are nearly constant, so the overall shape of the potential is not changed from the tree level (17), and the overall normalization is compensated by a suitable choice of the free parameter $\xi$. Thus, in this case the only effect is the lower bound on the Higgs boson mass, corresponding to $\lambda(\mu)$ becoming negative above some scale completely preventing inflation. In the prescription II, the logarithmic terms in (43) are significant, and the CMB predictions are modified, with large deviation from the tree-level results at low Higgs boson masses.

The lower bound on the Higgs mass can be intuitively understood from the following argument. If Higgs self-coupling $\lambda$ becomes zero (or negative) at any scale at or after inflation due to RG evolution, the second minimum of the RG-improved potential (44) develops at this scale, in addition to the ordinary minimum at zero (electroweak scale). This will stop the inflation or preheating in this minimum instead of the one we are living in at present. To a good precision in the Higgs mass, this bound can be obtained from the SM RG running of the coupling constants up to the scale $M_{P} / \xi$. As far as at these scales the logarithmic derivative of $\lambda$ is small, the exact scale which is used $\left(M_{P} / \xi\right.$ or $\left.M_{P} / \sqrt{\xi}\right)$ has a weak impact on the Higgs mass.

Careful analysis, involving the multi-loop renormalization running of the coupling constants between the electroweak and inflationary scales, and multi-loop relation between the $\overline{\mathrm{MS}}$ constants at the electroweak scale and physical observables (pole masses), was performed in $[63,64]$ (see also $[20,65,66]$ for previous results). The results (for the scale-invariant quantization I) give the lower bound on the Higgs mass

$$
m_{h}>\left(129.5+1.8 \frac{M_{t}-173.2 \mathrm{GeV}}{0.9 \mathrm{GeV}}-0.6 \frac{\alpha_{s}-0.1184}{0.0007} \pm 2\right) \mathrm{GeV}
$$

This is within errors of the actually measured Higgs mass, $m_{h} \simeq 125.5 \mathrm{GeV}[1,2]$.

Let us note that the bound on the Higgs mass depends on the way the model is quantized. For example, scale-invariant quantization (prescription I) just leads to the bound (46). At the same time, the choice II may lead to a stronger bound, occurring because of too small predicted $n_{s}$ (cf figure 4). Strictly speaking, this bound also relies on the possibility of relating the low-energy and high-scale physics, which relies on a relatively smooth transition through the scale $M_{P} / \xi$. Note here that to relax a bound on the Higgs mass in this way, one should have a significant difference between $\xi$ at high and low scales, with low scale $\xi$ being very large, significantly reducing the region of energies $\left(\mu<M_{P} / \xi\right)$, where the SM RG equations can be used.

Further improvement of the experimental situation is extremely interesting, as it may rule out the plain Higgs inflationary scenario or indicate the proper choice for the quantization (I versus II). To increase the precision of (46), one has to take into account higher order loop calculations, and, most importantly, to improve the experimental determination of the top mass $[63,67]$.

Evading the constraint (46) is possible by adding scalars to the theory, which provide additional positive contributions to the beta function of the Higgs self-coupling, thus loosening the bound $[68,69]$. 


\section{Variations of the Higgs inflation}

\subsection{Non-minimal derivative coupling}

The non-minimal coupling to the Ricci curvature is the lowest order operator that can be added to the minimally coupled action. This term does not lead to additional degrees of freedom in the theory. It turns out that it is possible to construct (uniquely) another term with the same property which is higher order in derivatives. $\ddagger$ Although normally higher order derivative terms lead to new degrees of freedom, the term

$$
\delta S_{\text {new }}=\int \mathrm{d}^{4} x \sqrt{-g}\left[-\omega^{2} G^{\mu \nu} \partial_{\mu} \Phi^{\dagger} \partial_{\nu} \Phi\right]
$$

has a specific cancellation and possesses not more than two time derivatives [70]. Here, $G^{\mu \nu}=R^{\mu \nu}-\frac{R}{2} g^{\mu \nu}$ is the Einstein tensor, and $\omega$ is the constant of dimensionality $M^{-2}$. This term has an effect of adding significant 'friction' to the scalar field background evolution in the expanding background. Actually, in the FRW background $G^{00} \sim H^{2}$. If $\omega H \gg 1$ the term (47) dominates the kinetic term. By the field redefinition $\bar{\Phi}=\Phi /(\omega H)$ during inflation we return the time derivative to the canonic normalization, which means an effective reduction of the quartic coupling constant $\lambda \rightarrow \bar{\lambda}=\lambda /(\omega H)^{4}$. This allows for the slow roll with $\lambda \sim 1$.

The CMB normalization gives [71] for the scale $\omega$ in (47)

$$
\omega^{-1}=\left(\left(24 \pi^{2} \Delta_{\mathcal{R}}^{2}\right)^{3} \frac{4}{243 \lambda N^{5}}\right)^{1 / 4} M_{P} \simeq 5 \times 10^{-8} \lambda^{-1 / 4} M_{P}
$$

The predictions for the CMB spectrum and tensor-to-scalar ratio at the tree level are (see figure 2)

$$
n_{s} \simeq 1-\frac{5}{3 N} \simeq 0.972, \quad r \simeq \frac{8}{3 N} \simeq 0.044 .
$$

The scale (48) required by CMB normalization is rather low, which could be expected as far as the operator (47) is of a higher order than the non-minimal coupling (1). Thus (47) is normally sub-leading for the HI compared to (1). Note, however, that applied to other inflationary models, the term in (47) can be important. An example is the inflation proceeding not along the modulus of the complex field $\Phi$, but along its angular direction (as in natural inflation with an axion) [72, 73]. In this case, the term (47) is relevant, while the non-minimal coupling (1) only rescales the overall normalization of the Planck mass without significantly modifying the shape of the potential.

Some analysis of the unitarity violation in this model can be found in [45]. Note that similar to the ordinary Higgs inflation, the cut-off scale is background dependent and becomes large at inflation.

\subsection{UV-completed Higgs inflation}

An important example is the perturbative attempt to provide a UV completion (see section 4) to the Higgs inflation which was suggested in [74]. This model provides the perturbative UV completion up to the Planck scale, however, at the cost of introducing additional parameters and modifying the connection between the inflationary and low-energy scales.

$\ddagger$ It is possible to add infinitely many terms without derivatives, like $h^{n} R$. However, their interest for the Higgs inflation is limited, as they lead to the Einstein frame potential decreasing at high field values, unless matched with the Jordan frame scalar potential $V \propto h^{2 n}[17]$. 
An additional scalar field $\sigma$ is introduced with the action

$$
\begin{aligned}
S_{J}=\int & \mathrm{d}^{4} x \sqrt{-g}\left[-\frac{\left(M^{2}+\xi_{\sigma} \sigma^{2}+2 \xi \Phi^{\dagger} \Phi\right)}{2} R+\frac{1}{2}\left(\partial_{\mu} \sigma\right)^{2}+\left|D_{\mu} \Phi\right|^{2}\right. \\
& \left.-\frac{\kappa}{4}\left(\sigma^{2}-\Sigma^{2}-2 \alpha \Phi^{\dagger} \Phi\right)^{2}-\lambda\left(\Phi^{\dagger} \Phi-\frac{v^{2}}{2}\right)^{2}\right]
\end{aligned}
$$

Here, the Higgs vacuum expectation value $v$ is assumed to be completely negligible for all inflationary and gravitational effects, while the vacuum value of the field $\sigma$ contributes significantly to the Planck mass $M_{P}^{2}=M^{2}+\xi_{\sigma} \Sigma^{2}$. In the limit $M=0$ the model of the $\sigma$ field behaves as an induced gravity model [26, 27]. In the limit $\alpha=0$ the $\sigma$ field is coupled with other fields only via gravity (or is mixed with all non-conformal terms after the conformal transformation to the Einstein frame), closely related to the model of [28] or $R^{2}$ gravity [33]. The non-minimal constant for the field $\sigma$ is assumed to dominate the Higgs nonminimal coupling, $\xi_{\sigma} \gg \xi$. In this limit, the flat inflationary direction in the Einstein frame is aligned mostly with the $\sigma$ field than with the Higgs field, and the CMB normalization now determines the constant $\xi_{\sigma}$ :

$$
\xi_{\sigma} \sqrt{\frac{\lambda+\kappa \alpha^{2}}{\kappa \alpha}} \simeq 5 \times 10^{4}
$$

(cf with (19)). The predictions for the spectral index and tensor-to-scalar ratio coincide with the predictions of the Higgs inflation (20) in the large $N$ limit (with probably slightly modified $N$ because of a different preheating mechanism). However, strictly speaking, the inflation is no longer governed by the Higgs field, but by the new field $\sigma$ with its own set of coupling constants $\xi_{\sigma}, \kappa$. In the argument of section 5, this UV completion leads to quadratic divergences from the point of view of the low-energy theory, i.e. the contributions form the mass of the $\sigma$ particle, thus removing the connection between the low-energy and inflationary regimes.

In the Higgs sector (and its interaction with gravity), the strong coupling problem does not arise because $\xi$ is now small. At the same time, the sector of the field $\sigma$ and gravity is close to the induced gravity limit of section 3.1.3 (i.e. $M^{2} \sim \xi_{\sigma} \Sigma^{2}$ ), where the inflationary potential is exactly (17), and has the cut-off scale $M_{P}$, instead of $M_{P} / \xi_{\sigma}$. Note that one can also think of the model as a perturbative completion of the HI model, where a new state $(\sigma)$ appears with the mass equal to the scale of the expected violation of the tree-level unitarity.

There were other attempts to construct the unitary completion by the modification of the kinetic interactions, see $[75,76]$.

\subsection{Higgs-dilaton theory}

Another variation of the induced gravity regime is used in the Higgs-dilaton models $[52,56,77-79]$. The model aims to create a completely scale-free theory, so an additional dilaton is introduced with the scalar part of the action

$S=\int \mathrm{d}^{4} x \sqrt{-g}\left[-\frac{\xi_{\sigma} \sigma^{2}+\xi h^{2}}{2} R+\frac{\left(\partial_{\mu} \sigma\right)^{2}}{2}+\frac{\left(\partial_{\mu} h\right)^{2}}{2}-\frac{\lambda}{4}\left(h^{2}-\frac{\alpha}{\lambda} \sigma^{2}\right)^{2}-\beta \sigma^{4}\right]$.

If $\beta=0$, then the scale invariance can be broken spontaneously in the flat space, leading to the generation of the planck mass $M_{P}^{2}=\xi_{\sigma} \sigma^{2}$. If one also assumes that the gravity satisfies the unimodular constraint $\operatorname{det}(g)=1$, the present-day cosmological constant effectively emerges in the theory. 
The model has a rather similar inflationary behaviour, but with increasing $\xi_{\sigma}$ the predictions change, with $n_{s}$ going out of the $2 \sigma$ allowed region for $\xi_{\sigma} \gtrsim 0.007$. The relation is present between the spectral index and the equation of state for the dark energy predicted by the model, which is an interesting relation between the properties of the early and late evolution of the Universe.

\subsection{Other variations}

The important class of models is the supergravity models leading to non-minimally coupled inflation. Note, that simple inclusion of non-zero $\xi$ into the supersymmetric model requires special formulation of the supergravity, as far as the traditional one leads to $\xi=0$. This modification was done in $[46,80-83]$. Note, that requirements of the stability of the inflationary potential mean that the low energy model can not be the simplest MSSM one, but is an extended NMSSM. The developed superconformal variation of supergravity allows for other non-minimally coupled inflationary models [84], leading to an interesting class of supersymmetric inflationary models. Higgs inflation with additional scalars can lead to models with a scalar cold dark matter [61, 68, 85-87]. Note also, that if arbitrary modifications of the kinetic term are allowed, more models leading, at least in the classical level, to the inflationary behaviour, e.g. [75, 88-90]

\section{Conclusions}

The Higgs inflationary model provides a nice and economical setup for the inflationary expansion of the Universe, without the introduction of any new particles beyond the already discovered SM ones (one still needs some new physics, for example, relatively light righthanded neutrinos, to explain neutrino oscillations, dark matter and baryon asymmetry of the Universe). The predictions of the model for the CMB are in excellent agreement with the latest observations. However, the theory is not UV complete, and although all the relevant calculations in the model are always performed at the momenta below the (backgrounddependent) scale, corresponding to the tree-level unitarity violation, taking into account the quantum corrections depends on the properties of the (yet unknown) UV completion, and may modify the model predictions. Scale invariance is the generic requirement for the UV completion.

The most interesting future experimental observations, that are important for the validity of the model, are the polarization of the CMB and the masses of the Higgs boson and top quark and the SM Higgs boson properties. Large $B$-mode polarization of the CMB, meaning a large tensor-to-scalar ratio, would mean a contradiction with the predictions of HI. Further improvement of the Higgs mass measurement together with the improvement on the top quark mass (possible, for example, on a lepton collider with the centre of mass energy above $350 \mathrm{GeV}$ ) will show if the SM vacuum is metastable, or can be stable and allow for the Higgs inflation.

\section{References}

[1] Chatrchyan S et al. (CMS Collaboration) 2012 Phys.Lett. B716 30-61 (arXiv:1207.7235)

[2] Aad G et al. (ATLAS Collaboration) 2012 Phys.Lett. B716 1-29 (arXiv:1207.7214)

[3] Ade P et al. (Planck Collaboration) 2013 (arXiv:1303.5082)

[4] Linde A D 1983 Phys. Lett. B129 177-181

[5] Kaiser D I 1995 Phys. Rev. D52 4295-4306 (arXiv:astro-ph/9408044) 
[6] Asaka T, Blanchet S and Shaposhnikov M 2005 Phys. Lett. B631 151-156 (arXiv:hep-ph/0503065)

[7] Asaka T and Shaposhnikov M 2005 Phys. Lett. B620 17-26 (arXiv:hep-ph/0505013)

[8] Boyarsky A, Ruchayskiy O and Shaposhnikov M 2009 Ann.Rev.Nucl.Part.Sci. 59 191-214 (arXiv:0901.0011)

[9] Birrell N D and Davies P C W 1982 Quantum Fields in Curved Space (Cambridge, UK: Univ. Pr.)

[10] Tsujikawa S and Gumjudpai B 2004 Phys. Rev. D69 123523 (arXiv:astro-ph/0402185)

[11] Makino N and Sasaki M 1991 Prog. Theor. Phys. 86 103-118

[12] Fakir R, Habib S and Unruh W 1992 Astrophys. J. 394396

[13] Tsujikawa S and Yajima H 2000 Phys.Rev. D62 123512 (arXiv:hep-ph/0007351)

[14] Kaiser D I 2010 Phys.Rev. D81 084044 (arXiv:1003.1159)

[15] Linde A 2008 Lect. Notes Phys. 738 1-54 (arXiv:0705.0164)

[16] Lerner R N and McDonald J 2010 JCAP 1004015 (arXiv:0912.5463)

[17] Park S C and Yamaguchi S 2008 JCAP 0808009 (arXiv:0801.1722)

[18] Kaiser D I, Mazenc E A and Sfakianakis E I 2013 Phys.Rev. D87 064004 (arXiv:1210.7487)

[19] Kaiser D I and Sfakianakis E I 2013 (arXiv:1304.0363)

[20] Bezrukov F and Shaposhnikov M 2009 JHEP 07089 (arXiv:0904.1537)

[21] Dutta S, Hagiwara K, Yan Q S and Yoshida K 2008 Nucl. Phys. B790 111-137 (arXiv:0705.2277)

[22] Salopek D S, Bond J R and Bardeen J M 1989 Phys. Rev. D40 1753

[23] Bezrukov F L and Shaposhnikov M 2008 Phys. Lett. B659 703-706 (arXiv:0710.3755)

[24] Bezrukov F and Gorbunov D 2011 Phys. Lett. B713 365 (arXiv:1111.4397)

[25] Bezrukov F and Gorbunov D 2013 JHEP 1307140 (arXiv:1303.4395)

[26] Zee A 1979 Phys. Rev. Lett. 42417

[27] Smolin L 1979 Nucl. Phys. B160 253

[28] Spokoiny B L 1984 Phys. Lett. B147 39-43

[29] Fakir R and Unruh W G 1990 Phys. Rev. D41 1783-1791

[30] Cooper F and Venturi G 1981 Phys.Rev. D24 3338

[31] Cervantes-Cota J and Dehnen H 1995 Phys.Rev. D51 395-404 (arXiv:astro-ph/9412032)

[32] Cervantes-Cota J and Dehnen H 1995 Nucl.Phys. B442 391-412 (arXiv:astro-ph/9505069)

[33] Starobinsky A A 1980 Phys. Lett. B91 99-102

[34] Gorbunov D and Panin A 2011 Phys.Lett. B700 157-162 (arXiv:1009.2448)

[35] Gorbunov D and Panin A 2012 Phys.Lett. B718 15-20 (arXiv:1201.3539)

[36] Gorbunov D and Tokareva A 2012 (arXiv:1212.4466)

[37] Bezrukov F, Gorbunov D and Shaposhnikov M 2009 JCAP 0906029 (arXiv:0812.3622)

[38] Garcia-Bellido J, Figueroa D G and Rubio J 2009 Phys. Rev. D79 063531 (arXiv:0812.4624)

[39] Bezrukov F, Magnin A, Shaposhnikov M and Sibiryakov S 2011 JHEP 1101016 (arXiv:1008.5157)

[40] Cornwall J M, Levin D N and Tiktopoulos G 1974 Phys. Rev. D10 1145

[41] Burgess C P, Lee H M and Trott M 2009 JHEP 09103 (arXiv:0902.4465)

[42] Barbon J L F and Espinosa J R 2009 Phys. Rev. D79 081302 (arXiv:0903.0355)

[43] Burgess C P, Lee H M and Trott M 2010 JHEP 07007 (arXiv:1002.2730)

[44] Hertzberg M P 2010 JHEP 11023 (arXiv:1002.2995)

[45] Atkins M and Calmet X 2011 Phys.Lett. B697 37-40 (arXiv:1011.4179)

[46] Ferrara S, Kallosh R, Linde A, Marrani A and Van Proeyen A 2011 Phys.Rev. D83 025008 (arXiv:1008.2942)

[47] Hertzberg M P 2012 JCAP 1208008 (arXiv:1110.5650)

[48] Barvinsky A O, Kamenshchik A Y and Starobinsky A A 2008 JCAP 0811021 (arXiv:0809.2104)

[49] De Simone A, Hertzberg M P and Wilczek F 2009 Phys. Lett. B678 1-8 (arXiv:0812.4946)

[50] Barvinsky A, Kamenshchik A, Kiefer C, Starobinsky A and Steinwachs C 2009 JCAP 0912003 (arXiv:0904.1698)

[51] Barvinsky A, Kamenshchik A Y, Kiefer C, Starobinsky A and Steinwachs C 2012 Eur.Phys.J. C72 2219 (arXiv:0910.1041) 
[52] Bezrukov F, Karananas G K, Rubio J and Shaposhnikov M 2013 Phys. Rev. D 87, 096001 (arXiv:1212.4148)

[53] Bezrukov F, Gorbunov D and Shaposhnikov M 2011 JCAP 1110001 (arXiv:1106.5019)

[54] Cheung C, Creminelli P, Fitzpatrick A L, Kaplan J and Senatore L 2008 JHEP 03014 (arXiv:0709.0293)

[55] Kallosh R, Linde A D, Linde D A and Susskind L 1995 Phys. Rev. D52 912-935 (arXiv:hep-th/9502069)

[56] Shaposhnikov M and Zenhausern D 2009 Phys. Lett. B671 162-166 (arXiv:0809.3406)

[57] Bezrukov F L, Magnin A and Shaposhnikov M 2009 Phys. Lett. B675 88-92 (arXiv:0812.4950)

[58] Weinberg S 2005 Phys. Rev. D72 043514 (arXiv:hep-th/0506236)

[59] George D P, Mooij S and Postma M 2012 JCAP 1211043 (arXiv:1207.6963)

[60] Englert F, Truffin C and Gastmans R 1976 Nucl. Phys. B117 407

[61] Lerner R N and McDonald J 2011 Phys.Rev. D83 123522 (arXiv:1104.2468)

[62] Mooij S and Postma M 2011 JCAP 1109006 (arXiv:1104.4897)

[63] Bezrukov F, Kalmykov M Y, Kniehl B A and Shaposhnikov M 2012 JHEP 1210140 (arXiv:1205.2893)

[64] Degrassi G, Di Vita S, Elias-Miro J, Espinosa J R, Giudice G F, Isidori G and Strumia A 2012 JHEP 1208098 (arXiv:1205.6497)

[65] Espinosa J R, Giudice G F and Riotto A 2008 JCAP 0805002 (arXiv:0710.2484)

[66] Elias-Miro J, Espinosa J R, Giudice G F, Isidori G, Riotto A and Strumia A 2012 Phys.Lett. B709 222-228 (arXiv:1112.3022)

[67] Alekhin S, Djouadi A and Moch S 2012 Phys.Lett. B716 214-219 (arXiv:1207.0980)

[68] Clark T, Liu B, Love S and ter Veldhuis T 2009 Phys.Rev. D80 075019 (arXiv:0906.5595)

[69] Elias-Miro J, Espinosa J R, Giudice G F, Lee H M and Strumia A 2012 JHEP 1206031 (arXiv:1203.0237)

[70] Germani C and Kehagias A 2010 Phys. Rev. Lett. 105011302 (arXiv:1003.2635)

[71] Germani C and Kehagias A 2010 JCAP 1005019 (arXiv:1003.4285)

[72] Germani C and Kehagias A 2011 Phys.Rev.Lett. 106161302 (arXiv:1012.0853)

[73] Germani C and Watanabe Y 2011 JCAP 1107031 (arXiv:1106.0502)

[74] Giudice G F and Lee H M 2011 Phys.Lett. B694 294-300 (arXiv:1010.1417)

[75] Lerner R N and McDonald J 2010 Phys.Rev. D82 103525 (arXiv:1005.2978)

[76] Lerner R N and McDonald J 2012 JCAP 1211019 (arXiv:1112.0954)

[77] Shaposhnikov M and Zenhausern D 2009 Phys. Lett. B671 187-192 (arXiv:0809.3395)

[78] Garcia-Bellido J, Rubio J and Shaposhnikov M 2012 Phys.Lett. B718 507-511 (arXiv:1209.2119)

[79] Garcia-Bellido J, Rubio J, Shaposhnikov M and Zenhausern D 2011 Phys.Rev. D84 123504 (arXiv:1107.2163)

[80] Arai M, Kawai S and Okada N 2012 Phys.Rev. D86 063507 (arXiv:1112.2391)

[81] Arai M, Kawai S and Okada N 2011 Phys.Rev. D84 123515 (arXiv:1107.4767)

[82] Ferrara S, Kallosh R, Linde A, Marrani A and Van Proeyen A 2010 Phys.Rev. D82 045003 (arXiv:1004.0712)

[83] Kallosh R and Linde A 2010 JCAP 1011011 (arXiv:1008.3375)

[84] Kallosh R and Linde A 2013 JCAP 1306027 (arXiv:1306.3211)

[85] Lerner R N and McDonald J 2009 Phys.Rev. D80 123507 (arXiv:0909.0520)

[86] Okada N and Shafi Q 2011 Phys.Rev. D84 043533 (arXiv:1007.1672)

[87] Gong J O, Lee H M and Kang S K 2012 JHEP 1204128 (arXiv:1202.0288)

[88] Kamada K, Kobayashi T, Yamaguchi M and Yokoyama J 2011 Phys.Rev. D83 083515 (arXiv:1012.4238)

[89] Kamada K, Kobayashi T, Takahashi T, Yamaguchi M and Yokoyama J 2012 Phys.Rev. D86 023504 (arXiv:1203.4059)

[90] Nakayama K and Takahashi F 2011 JCAP 1102010 (arXiv:1008.4457) 